\title{
A Biochemical Characterization of Histidine-Dependent Mutants of Staphylococcus aureus
}

\author{
By W. E. KLOOS and P. A. PATTEE \\ Department of Bacteriology, Iowa State University, Ames, Iowa, U.S.A.
}

(Received 21 October 1964)

\begin{abstract}
SUMMARY
Over 100 histidine-dependent mutants were isolated from strain 655 of Staphylococcus aureus. Paper chromatography was used to differentiate the mutants into classes according to their ability to accumulate Paulypositive imidazoles. Classes $\boldsymbol{E}$ and $\boldsymbol{G}$ did not accumulate any detectable Pauly-positive imidazoles. Mutants of class $A$ accumulated 5-amino-1ribosyl-4-imidazolecarboxamide, mutants of class $B$ accumulated imidazoleglycerol, and mutants of class $C$ accumulated imidazoleacetol. Mutants of class $D$ accumulated histidinol and were incapable of utilizing exogenous L-histidinol. A comparison of the accumulations obtained from mutants of $S$. aureus with accumulations obtained from previously characterized mutants of Salmonella typhimurium indicates that a similar, if not identical, pathway for the biosynthesis of L-histidine is used by these two species.
\end{abstract}

\section{INTRODUCTION}

Vogel, Davis \& Mingioli (1951) isolated L-histidinol from a histidine-dependent mutant of Escherichia coli and observed that this compound could support the growth of another histidine-dependent mutant. A chromatographic analysis of imidazoles accumulated by histidine-dependent mutants of Neurospora crassa indicated the existence of at least four classes of biotypes (Haas, Mitchell, Ames \& Mitchell, 1952). These mutants accumulated Pauly-positive imidazoles which were tentatively identified as imidazoleglycerol (IG), imidazoleacetol (IA) and histidinol (Ames, Mitchell \& Mitchell, 1953). Subsequent studies of these mutants revealed that they accumulated the phosphate esters imidazoleglycerol phosphate (IGP), imidazoleacetol phosphate (IAP) and histidinol phosphate (HP) in addition to the unphosphorylated compounds (Ames \& Mitchell, 1955). None of the imidazoles accumulated by these histidine-dependent mutants was capable of relieving the histidine requirement of other mutants. Hartman (1956) identified four classes of histidine-dependent mutants of Salmonella typhimurium. $A$ mutants were distinguished by failure to accumulate Pauly-positive imidazoles. $B$ mutants accumulated IG and IGP. $C$ mutants accumulated IA, IAP, imidazolelactic acid (ILA) and smaller quantities of IG and IGP. $D$ mutants accumulated histidinol, HP, and smaller quantities of IA, IAP, ILA, IG and IGP. Of the imidazoles accumulated, only histidinol and ILA supported the growth of mutants blocked at earlier reactions, while the other imidazoles were considered to be either impermeable to the cell membrane (e.g. phosphate esters) or not actual precursors of L-histidine (e.g. IG and IA). Genetic complementation and quantitative cell-free enzyme analyses 
of various $\boldsymbol{A}$ mutants showed the presence of at least four different phenotypic classes, designated $E, A, H$ and $F$ (Hartman, Loper \& Serman, 1960). The genetic studies by these workers indicated the presence of another phenotypic class of mutants, designated $G$, which like the $\boldsymbol{A}$ mutants did not accumulate Paulypositive imidazoles. However, unlike the $\boldsymbol{A}$ mutants, the $\boldsymbol{G}$ mutants were located adjacent to the $D$ locus and thus at a considerable distance from the $E, A, H$ and $F$ loci. Smith \& Ames (1964) separated the $E$ mutants into two distinct classes, $E$ and I. The newly designated $E$ mutants accumulated the Bound Bratton-Marshall compound BBM I, whereas $I$ mutants did not accumulate any Bound BrattonMarshall compounds. A more complete understanding of the pathway used for the biosynthesis of L-histidine has been possible as a result of the studies of specific enzyme activities in cell-free extracts of various histidine-dependent mutants of S. typhimurium (Ames \& Hartman, 1962; Smith \& Ames, 1964).

The present report describes a paper-chromatographic analysis of Pauly-positive imidazoles accumulated by histidine-dependent mutants of Staphylococcus aureus, and the degree of similarity of these compounds to those obtained from previously characterized histidine-dependent mutants of Salmonella typhimurium. Information about the ability of histidine-dependent mutants of Staphylococcus aureus to utilize various imidazoles in place of exogenous L-histidine also is presented. The companion paper (Kloos \& Pattee, 1965) describes the genetic analysis of these mutants of $S$. aureus.

\section{METHODS}

Media. In addition to commercially available dehydrated media, two defined media were used in this work. Studies involving mutants of Salmonella typhimurium were made with the defined medium (' $E$ ' broth) of Vogel \& Bonner (1956) supplemented with various concentrations of L-histidine (monohydrate; California Corporation for Biochemical Research (Calbiochem), Los Angeles, Calif., U.S.A.). The defined medium (S broth) used for the study of mutants of Staphylococcus aureus consisted of the following ingredients: $\mathrm{K}_{2} \mathrm{HPO}_{4}, 7 \mathrm{~g}$.; $\mathrm{KH}_{2} \mathrm{PO}_{4}, 2$ g.; sodium citrate. $5 \mathrm{H}_{2} \mathrm{O}, 0.5 \mathrm{~g}$.; $\mathrm{MgSO}_{4} \cdot 7 \mathrm{H}_{2} \mathrm{O}, 0 \cdot 1$ g., $\left(\mathrm{NH}_{4}\right)_{2} \mathrm{SO}_{4}, 1 \mathrm{~g}$.; glucose, 4 g.; glycine, $50 \mathrm{mg}$; L-glutamic acid, $100 \mathrm{mg}$; L-aspartic acid, $90 \mathrm{mg}$.; L-serine, $30 \mathrm{mg}$; Lmethionine, $3 \mathrm{mg}$; L-cystine, $20 \mathrm{mg}$; L-alanine, $60 \mathrm{mg}$.; L-lysine. $\mathrm{HCl}, 50 \mathrm{mg}$.; L-arginine. $\mathrm{HCl}, 50 \mathrm{mg}$; L-threonine, $30 \mathrm{mg}$; L-phenylalanine, $40 \mathrm{mg}$; ; L-tryptophan, $10 \mathrm{mg}$.; L-leucine, $90 \mathrm{mg}$.; L-isoleucine, $30 \mathrm{mg}$; L-proline, $80 \mathrm{mg}$.; L-valine, $80 \mathrm{mg}$.; nicotinic acid, $1 \mathrm{mg}$; ; thiamine. $\mathrm{HCl}, 1 \mathrm{mg}$; de-ionized water, $1000 \mathrm{ml}$. Glucose was added aseptically after sterilization of the defined medium. The concentration of either L-histidine or L-histidinol (dihydrochloride; Mann Research Laboratories, New York, N.Y.) added to S broth was varied according to requirements. Defined medium agar (S agar) was prepared by adding $1.5 \%(\mathrm{w} / \mathrm{v})$ Noble agar (Difco) to $\mathrm{S}$ broth. All glassware used with the defined media was cleaned in chromic+sulphuric acid mixture and rinsed thoroughly with deionized water before use.

Bacterial strains. The histidine-dependent mutants hisE-11, hisF-41, hisA-3, hisH-107, hisB-456, hisC-2, hisD-494 and hisG-46 of Salmonella typhimurium were obtained from Dr P. E. Hartman (The Johns Hopkins University); these mutants were described by Hartman et al. (1960). All histidine-dependent mutants of 
Staphylococcus aureus were obtained from either strain 655 (Pattee \& Baldwin, 1962) or a penicillinase-deficient mutant of this strain. All strains of bacteria were maintained at $4^{\circ}$ on Brain Heart Infusion (BHI; Difco) agar slopes in screwcapped tubes. The inocula for all experiments were obtained from subcultures of these stocks, previously prepared on BHI agar slopes incubated at $37^{\circ}$ for $12 \mathrm{hr}$ and then stored at $4^{\circ}$. These subcultures were replaced at weekly intervals. In all instances, the shaking of cultures was performed with a Burrell Wrist-Action Shaker, (2223 Fifth Avenue, Pittsburgh, Pa, U.S.A.) with the arms mounted over water baths. All growth experiments were conducted at $37^{\circ}$.

Isolation of mutants. Sixteen histidine-dependent mutants were obtained from Staphylococcus aureus strain 655 during preliminary studies. The majority of the mutants, however, were derived from a single penicillinase-deficient mutant of strain 655 by means of ultraviolet irradiation and the following modification of the penicillin selection technique of Davis (1949) and Lederberg (1950). A $12 \mathrm{hr}$ BHI agar slope culture of the parent strain was suspended in $5 \mathrm{ml}$. saline, and $0 \cdot 1 \mathrm{ml}$. of this suspension added to each of two tubes containing $5 \mathrm{ml}$. $\mathrm{S}$ broth. The tubes were shaken for $12 \mathrm{hr}$ at a shaker setting of 7 . At the end of this period, the cultures were centrifuged and the packed cocci uniformly suspended in $5 \mathrm{ml}$. saline. The suspensions were mixed in an open flat-bottomed Petri dish (100 mm. diam.) and exposed for $45 \mathrm{sec}$. and with constant agitation to radiation from a 30-Watt G. E. Germicidal bulb at a distance of $85 \mathrm{~cm}$. Two tubes, each containing $5 \mathrm{ml}$. S broth, were inoculated with $\mathbf{0} \cdot \mathbf{1} \mathrm{ml}$. samples of the u.v.-irradiated suspension. Both tubes were shaken at a shaker setting of 7 , and penicillin $\mathrm{G}$ ( 1 unit $/ \mathrm{ml}$.) added after the fifth hour. After further incubation for $26 \mathrm{hr}$ during which shaking was continued, the cultures were centrifuged, the cocci washed once with saline and resuspended in $3 \mathrm{ml}$. saline. Dilutions of 1/10 and 1/100 of the final coccal suspension were prepared, and 0.1 ml. samples spread over the surfaces of Trypticase Soy Agar(TSA; Baltimore Biological Co.) plates. These plates were incubated for $24 \mathrm{hr}$, after which the colonies were inoculated by the replica plating technique (Lederberg \& Lederberg, 1952) on to TSA and S agar plates. After incubation of the replica plates for $24 \mathrm{hr}$, those colonies which grew well on TSA but did not do so on S agar were subcultured for further study. All isolates were typed with bacteriophages $29,52,52 \mathrm{~A}$, $79,80,81,6,7,83,42 \mathrm{~B}, 47 \mathrm{C}, 47,53,54,70,73,75,77$ and $44 \mathrm{~A}$ of the International Typing Series. These phages and the phage-typing procedure were described by Pattee \& Baldwin (1961). The growth response of each isolate to exogenous Lhistidine then was determined by spreading about $10^{7}$ cocci of the isolate over the surface of an $\mathrm{S}$ agar plate. Several crystals of L-histidine were placed at the centre of the plate, which was incubated for $24 \mathrm{hr}$ and examined for evidence of growth. Strains which did not grow on $S$ agar except in the presence of exogenous L-histidine were maintained for further study.

Designation of mutants. Based upon the nature of their primary accumulations of Pauly-positive imidazoles, the mutants of Staphylococcus aureus were placed in classes as follows: his $A$ mutants accumulate 5-amino-1-ribosyl-4-imidazolecarboxamide (AIC-R); hisB mutants accumulate imidazoleglycerol (IG); hisC mutants accumulate imidazoleacetol (IA); hisD mutants accumulate histidinol. The hisE and hisG mutants did not accumulate Pauly-positive imidazoles, and were differentiated on the basis of their chromosomal position (Kloos \& Pattee, 1965). 
The mutants have been designated according to the scheme of Hartman et al. (1960); for example, mutant hisAa-83 is the 83rd histidine-dependent mutant isolated, accumulates AIC-R, and possesses a mutant site in the $a$ complementation unit of the $A$ gene (Kloos \& Pattee, 1965).

Methods of growth stimulation. All histidine-dependent mutants of Staphylococcus aureus were examined for their ability to utilize exogenous L-histidinol in place of L-histidine by the same methods as used to determine the histidinedependent nature of the mutants. Mutants hisAa-11, hisAb-1, hisAb-20, hisD-8 and $h i s G b-15$ were also tested for their ability to grow on $\mathrm{S}$ agar containing either histidinol, IG, IGP, IA, IAP, ILA or HP. These imidazoles were obtained from lyophilized supernatant fluids of cultures of appropriate histidine-dependent mutants of Salmonella typhimurium by preparative thin-layer chromatography.

Preparation of cultures for chromatographic analysis. To study the accumulation of Pauly-positive imidazoles by histidine-dependent mutants of Staphylococcus aureus, it was necessary to examine several variables in growth conditions. The results of this preliminary work led to adoption of the following procedure. A $12 \mathrm{hr}$ BHI agar slope culture of a mutant was used to inoculate $5 \mathrm{ml}$. S broth containing $20 \mu \mathrm{g}$. L-histidine $/ \mathrm{ml}$. The initial coccal concentration of this culture, referred to hereafter as the adaptation culture, was about $1 \times 10^{7} \mathrm{cocci} / \mathrm{ml}$. The culture was shaken at a shaker setting of 3 for $12 \mathrm{hr}$. One-tenth $\mathrm{ml}$. of the adaptation culture was then transferred to $5 \mathrm{ml}$. S broth containing $2 \cdot 0 \mu \mathrm{g}$. L-histidine or L-histidinol $/ \mathrm{ml}$. This culture, referred to hereafter as the de-repression culture, was prepared with $\mathrm{L}$ histidine for the study of the parent strain and $D$ mutants, these mutants being incapable of utilizing exogenous L-histidinol. The de-repression cultures were shaken at a shaker setting of $\mathbf{3}$ for $\mathbf{7 2} \mathrm{hr}$, after which the cocci were sedimented by centrifugation and the supernatant fluids lyophilized. The dried residue was then reconstituted in $0.2 \mathrm{ml}$. de-ionized water and analysed by ascending paper chromatography. Lyophilized supernatant fluids of cultures of Salmonella typhimurium were prepared in the manner described above, using ' $\mathbf{E}$ ' broth supplemented with $20 \mu \mathrm{g}$. L-histidine $/ \mathrm{ml}$. in the adaptation cultures, and $3.0 \mu \mathrm{g}$. L-histidine $/ \mathrm{ml}$. in the de-repression cultures.

Chromatography. Pauly-positive imidazoles were identified by ascending paper chromatography. The solvents used for the development of chromatograms were: (a) tert-butyl alcohol $+50 \%$ formic acid in water ( $7+3$ by vol., Ames \& Mitchell, 1955); (b) $n$-butyl alcohol saturated with $2 \mathrm{~N}-\mathrm{NH}_{3}$ (Block, Durrum \& Zweig, 1958); (c) $n$-propanol $+0 \cdot 2 \mathrm{~N}-\mathrm{NH}_{3}(3+1$ by vol., Ames \& Mitchell, 1952). Chromatograms were prepared on Whatman 3 MM filter paper, $0.03 \mathrm{ml}$. of concentrated supernatant fluid being applied as a spot on the origin. Three chromatograms of each mutant were prepared, to permit three solvent systems to be used in development. After each chromatogram was completely dry, it was developed in one of the solvents until the solvent front was $15 \mathrm{~cm}$. from the origin (about $30 \mathrm{~min}$ ). Each chromatogram then was dried for at least $2 \mathrm{hr}$ at room temperature, after which it was sprayed with diazo sulphanilic acid reagent followed by a $5 \%(w / v)$ solution of $\mathrm{Na}_{2} \mathrm{CO}_{3}$ according to the modified Pauly technique of Ames \& Mitchell (1952).

Chemical standards used in the chromatographic analysis were L-histidine, L-histidinol, L-tyrosine, 5-amino-4-imidazolecarboxamide (AIC; Calbiochem), 
AIC-R (Calbiochem), imidazoleglycerol, and imidazolelactic acid. The last two imidazoles were provided by Dr P. E. Hartman. In addition to the chemical standards, lyophilized and reconstituted supernatant fluids from cultures of histidine-dependent mutants of Salmonella typhimurium were used as sources of known imidazoles.

\section{RESULTS}

Isolation of mutants. A total of 85 histidine-dependent mutants were obtained from strain 655 of Staphylococcus aureus by the penicillin selection technique. These mutants represented about $0.26 \%$ of the total number of colonies recovered on TSA agar. An additional 75 mutants requiring exogenous purines, pyrimidines or vitamins were also obtained, these nutrients being absent from $S$ broth and $S$ agar.

Growth stimulations. With the exception of the $D$ mutants, L-histidinol stimulated all histidine-dependent mutants of Staphylococcus aureus. Mutant hisAaD-63, which accumulated AIC-R, was not stimulated by L-histidinol. When added to de-repression cultures at concentrations of $10 \mu \mathrm{g} . / \mathrm{ml}$. or greater, L-histidinol inhibited the growth of those mutants of $S$. aureus which were capable of utilizing this compound. Growth of these mutants on S agar also was inhibited by L-histidinol, as evidenced by the zones of growth inhibition surrounding the area where crystalline $L$-histidinol was deposited. All mutants of $S$. aureus tested (hisAa-11, hisAb-1, hisAb-20, $h i s D-8, h i s G b-15)$ did not grow on $\mathrm{S}$ agar in the presence of either IG, IGP, IA, IAP, ILA or HP obtained from mutants of Salmonella typhimurium. With the exception of mutant hisD-8, these mutants were stimulated by histidinol obtained from mutant hisD-494 of $S$. typhimurium.

Accumulations. The solvents and spray reagents which were used in the final characterization of imidazoles, and the $R_{F}$ values and colours of these imidazoles are given in Table 1. The parent strain and all the histidine-dependent mutants of Staphylococcus aureus accumulated a compound which was indistinguishable from L-tyrosine, and which interfered with the detection of IG. These two compounds were most successfully resolved by means of the $n$-butanol + ammonia solvent system. The colour reaction of L-tyrosine also was weak when this solvent system was used. None of the solvent systems used was capable of adequately resolving IA from histidinol since both of these compounds showed considerable trailing and possessed similar $R_{F}$ values. However, a colour distinction between IA (red) and histidinol (yellow) was obtained with a sulphanilamide spray reagent (Block et al. 1958). The phosphorylated imidazoles IAP, IGP and HP could not be identified in supernatant fluids of cultures of $S$. aureus because of their low $R_{F}$ values, which placed them in an area of non-specific material and pigments near the origin.

The imidazoles accumulated by each histidine-dependent mutant of Staphylococcus aureus are shown in Table 2. Mutants his-26, his-28, his-53, his-57, his-67, $h i s-86$, his-92, his-97 and his-102 grew poorly, did not accumulate detectable quantities of Pauly-positive imidazoles and were excluded from the chromatographic analysis. In addition to the primary accumulations, secondary accumulations were detected in supernatant fluids of cultures of those mutants which exhibited good growth. Although many of the $D$ mutants may have accumulated small amounts of IA, this compound would not have been detectable in the presence of histidinol, the primary accumulation product of all $D$ mutants. The parent strain normally accumulated small amounts of IG and histidine, although only small 


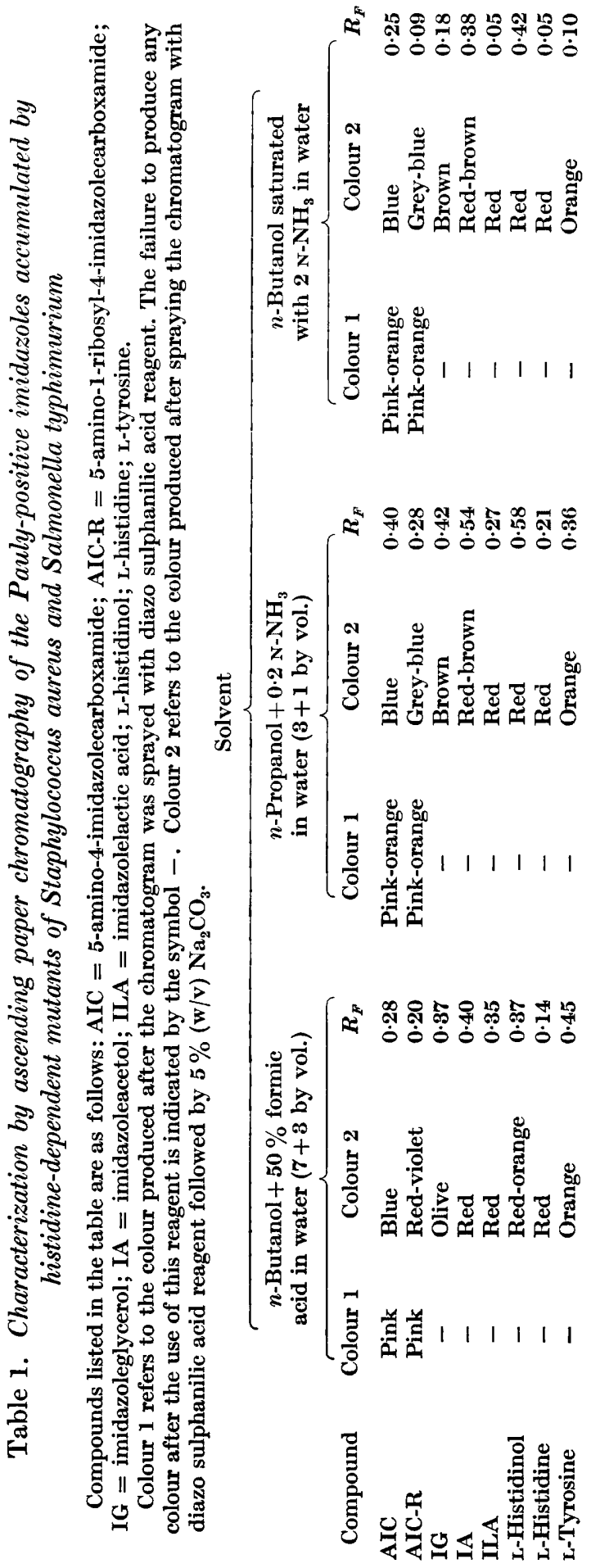




\section{Table 2. Some characteristics of histidine-dependent mutants of}

\section{Staphylococcus aureus}

Column I. Mutant is designated according to the scheme of Hartman, Loper \& Serman (1960). Column II. The number of prototrophic colonies recovered on $S$ agar from a $0.1 \mathrm{ml}$. sample of a $72 \mathrm{hr}$ de-repression culture. TMTC $=$ too many colonies to count. Column III. Growth responses on $\mathrm{S}$ agar containing several crystals of L-histidinol are recorded as negative $(-)$, or stimulation $(+)$. Column IV. Accumulations: $0=$ no Pauly-positive imidazoles detected; $1=5$-amino-1-ribosyl-4-aminoimidazolecarboxamide; $2=$ imidazoleglycerol; $3=$ imidazoleacetol; $5=$ histidinol.

\begin{tabular}{|c|c|c|c|c|c|c|c|}
\hline$\underset{\text { Mutant }}{\text { I }}$ & $\begin{array}{l}\text { II } \\
\text { Proto- } \\
\text { trophic } \\
\text { colonies } \\
\text { (no.) }\end{array}$ & $\begin{array}{c}\text { III } \\
\text { Growth } \\
\text { on } \\
\text { L- }^{-} \\
\text {histidinol }\end{array}$ & $\begin{array}{c}\text { IV } \\
\text { Accumu- } \\
\text { lations }\end{array}$ & $\underset{\text { Mutant }}{\text { I }}$ & $\begin{array}{c}\text { II } \\
\text { Proto- } \\
\text { trophic } \\
\text { colonies } \\
\text { (no.) }\end{array}$ & $\begin{array}{c}\text { III } \\
\text { Growth } \\
\text { on } \\
\text { L- } \\
\text { histidinol }\end{array}$ & $\begin{array}{c}\text { IV } \\
\text { Accumu- } \\
\text { lations }\end{array}$ \\
\hline$h i s A b-1$ & 0 & + & 1 & $h i s D-52$ & o & - & 5 \\
\hline$h i s B-2$ & 1 & + & 1,2 & hisD-54 & 0 & - & 1,5 \\
\hline hisD-3 & 0 & - & 5 & hisD-55 & $\mathbf{0}$ & - & 5 \\
\hline$h i s A b-5$ & 2 & + & 1 & hisC $=56$ & 0 & + & 1,3 \\
\hline $\operatorname{his} A b-6$ & 0 & + & 1 & hisD-58 & o & - & 5 \\
\hline $\operatorname{his} A b-7$ & 0 & + & 1 & hisD-59 & $\mathbf{0}$ & - & 1,5 \\
\hline$h i s D-8$ & 0 & - & $1,2,5$ & $h i s D-60$ & 0 & - & 5 \\
\hline hisGb-9 & 0 & + & 0 & hisD-61 & 0 & - & 5 \\
\hline $\operatorname{his} A b-10$ & 0 & + & 1 & hisGa-62 & 7 & + & 0 \\
\hline his $A a-11$ & 0 & + & 1 & his AaD-63 & 0 & - & 1 \\
\hline his $A b-12$ & 0 & + & 1 & $\operatorname{his} A-64$ & TMTC & + & 1 \\
\hline hisGb-13 & 0 & + & $\mathbf{0}$ & $h i s G-65$ & TMTC & + & 0 \\
\hline hisC-14 & 0 & + & $1,2,3$ & hisC-66 & TMTC & + & $1,2,3$ \\
\hline hisGb-15 & 1 & + & o & $h i s D-68$ & $\mathbf{5}$ & - & 5 \\
\hline hisD-16 & TMTC & - & 5 & hisD-69 & $\mathbf{0}$ & - & 5 \\
\hline his $A b-17$ & 1 & + & 1 & hisD-70 & 0 & - & 5 \\
\hline hisD-18 & $\mathbf{0}$ & - & 5 & hisD-71 & $\mathbf{0}$ & - & 5 \\
\hline his Ab-19 & 0 & + & 1 & hisD-72 & o & - & 1,5 \\
\hline his $A b-20$ & 0 & + & 1 & hisC-73 & 5 & + & 1,3 \\
\hline hisC-23 & 0 & + & 1,3 & hisGa-74 & 10 & + & 0 \\
\hline hisD-24 & 2 & - & 5 & hisGa-75 & 0 & + & $\mathbf{0}$ \\
\hline hisD:25 & 0 & - & $1,2,5$ & his $A c-76$ & 0 & $t$ & 1 \\
\hline$h i s B-27$ & 0 & + & 1,2 & his $A-7 \gamma$ & 78 & + & 1 \\
\hline$h i s G-29$ & TMTC & + & 0 & hisG-78 & 100 & + & 0 \\
\hline hisG-30 & TMTC & + & $\mathbf{0}$ & $h i s A a-79$ & 0 & + & 1 \\
\hline his $A a-31$ & 0 & + & 1 & his $A-80$ & o & + & 1 \\
\hline hisD-32 & 0 & - & 1,5 & $h i s D-81$ & 0 & - & 5 \\
\hline hisD.33 & 0 & - & 1,5 & hisD-82 & TMTC & - & 5 \\
\hline$h i s G-34$ & TMTC & + & 0 & his Aa-83 & 0 & + & $\mathbf{1}$ \\
\hline$h i s G-35$ & TMTC & + & $\mathbf{0}$ & hisD-84 & $\mathbf{0}$ & - & 5 \\
\hline hisD-36 & o & - & 5 & hisD-85 & o & - & 5 \\
\hline hisG-37 & TMTC & + & 0 & $h i s G-87$ & 263 & + & $\mathbf{0}$ \\
\hline hisGb-38 & 0 & + & $\mathbf{0}$ & hisD-88 & TMTC & - & 5 \\
\hline hisG-39 & TMTC & + & 0 & hisG-89 & 620 & + & 0 \\
\hline hisGa-40 & 0 & + & $\mathbf{0}$ & hisC-90 & 124 & + & 1,3 \\
\hline hisB-41 & 108 & + & 1,2 & hisC-91 & 0 & + & $1,2,3$ \\
\hline$h i s D-42$ & 0 & - & 5 & hisE-93 & 2 & + & 0 \\
\hline$h i s D-43$ & 0 & - & 5 & $h i s G-94$ & 410 & + & $\mathbf{0}$ \\
\hline hisD-44 & 0 & - & 5 & hisG-95 & TMTC & + & $\mathbf{0}$ \\
\hline$h i s G-45$ & 6 & + & 0 & hisC-96 & 65 & + & $\mathbf{3}$ \\
\hline$h i s D-46$ & 0 & - & 5 & hisD-98 & o & - & 5 \\
\hline$h i s D-47$ & 0 & - & 5 & hisGb-99 & 3 & + & o \\
\hline$h i s C-48$ & 0 & + & $1,2,3$ & hisD-100 & o & - & $\mathbf{5}$ \\
\hline$h i s E-49$ & $\mathbf{0}$ & + & 0 & hisC-101 & 120 & + & 3 \\
\hline$h i s C-50$ & 0 & + & 1,3 & hisD-103 & TMTC & - & 5 \\
\hline$h i s D-51$ & 0 & - & $1,2,5$ & hisD-104 & 0 & - & 5 \\
\hline
\end{tabular}


amounts of histidinol were detected when supernatant fluids were prepared from unshaken cultures.

A comparison of the imidazoles accumulated by representative mutants of Staphylococcus aureus and Salmonella typhimurium is shown in Fig. 1. One difference observed between mutants of these two species was the accumulation of AIC-R by $A$ mutants of $S$. aureus and the accumulation of AIC by the corresponding $E, A, H$ and $F$ mutants of $S$. typhimurium. Small amounts of ILA were accumulated by $C$ mutants of $S$.typhimurium but not by $C$ mutants of $S$. aureus. Also, mutants of $S$. typhimurium invariably produced greater quantities of accumulations than did mutants of $S$. aureus.

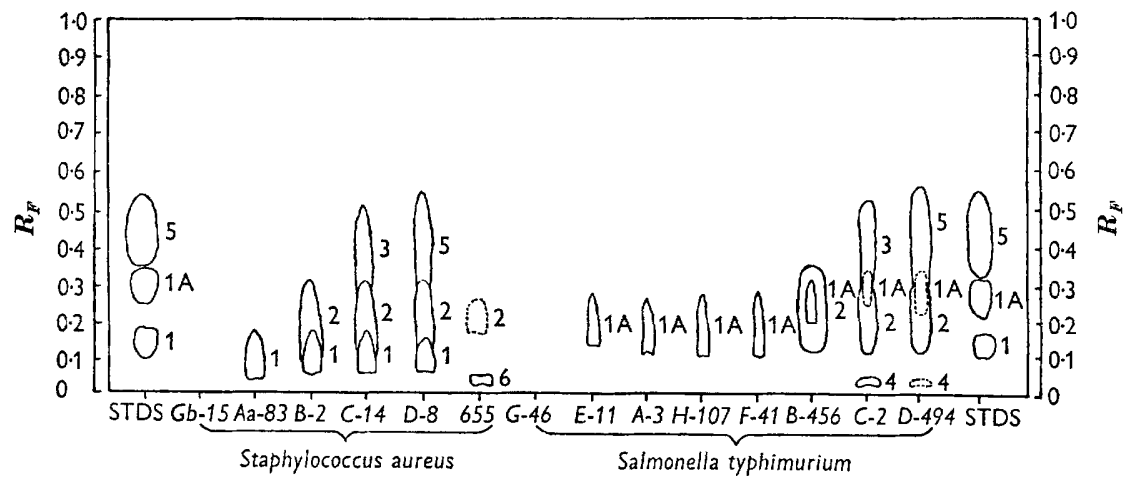

Fig. 1. Tracing of a chromatogram of Pauly-positive imidazoles accumulated by histidine-dependent mutants of Staphylococcus aureus and Salmonella typhimurium. The chromatogram was prepared on Whatman no. 3 MM filter paper and developed in $n$ butanol saturated with $2 \mathrm{~N}-\mathrm{NH}_{3}$ solution. Imidazoles were detected by spraying the chromatogram with diazo sulphanilic acid reagent followed by $5 \%(w / v) \mathrm{Na}_{2} \mathrm{CO}_{3}$. Imidazoles were identified as follows $1=5$-amino-1-ribosyl-4-imidazolecarboxamide; $1 \mathrm{~A}=5$-amino-4-imidazolecarboxamide; 2 = imidazoleglycerol; 3 = imidazoleacetol; $4=$ imidazolelactic acid; $5=$ histidinol; $6=$ histidine. The strains of $S$. aureus and $S$. typhimurium from which the imidazoles were obtained and the chemical standards (STDS) are indicated below the origin.

\section{DISCUSSION}

The pathway used by Salmonella typhimurium for the biosynthesis of L-histidine has been studied in detail (Ames, Garry \& Herzenberg, 1960; Ames, Martin \& Garry, 1961 ; Smith \& Ames, 1964) and has been proposed to be as follows:

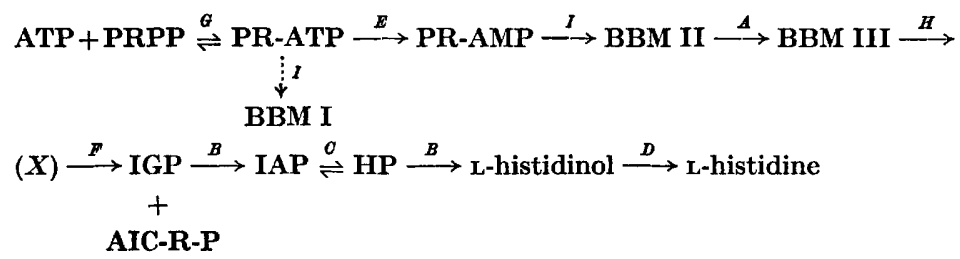

Based upon their failure to accumulate Pauly-positive imidazoles, the $G$ and $E$ mutants of Staphylococcus aureus are believed to correspond to the $\boldsymbol{G}$ and $I$ mutants, respectively, of Salmonella typhimurium. Hartman et al. (1960) reported that $E$, 
$A, H$ and $F$ mutants of $S$. typhimurium did not accumulate Pauly-positive imidazoles. In the present study, lyophilization of the supernatant fluids of cultures of these mutants permitted the detection of a compound which was indistinguishable from AIC. It is possible that AIC and AIC-R, the latter compound being accumulated by $A$ mutants of $S$. aureus, could be derived as cleavage products of the BBM compounds. Both IG and IA, accumulated by $B$ and $C$ mutants of both species, are believed to be derived from their corresponding phosphate esters (Haas et al. 1952; Hartman, 1956). Histidinol, accumulated by $D$ mutants of both species, is believed to be the only actual intermediate of L-histidine biosynthesis which has been identified in the culture fluids. This conclusion is supported by the observation that histidinol was the only imidazole among those accumulated by mutants of $S$. typhimurium which was utilized by mutants of $S$. aureus. The remaining imidazoles which were accumulated by mutants of $S$. typhimurium did not relieve the histidine requirement of mutants of $S$. aureus because they were either impermeable to the cell (i.e. phosphate esters: Hass et al. 1952; Hartman, 1956) or not actual intermediates in the pathway of histidine biosynthesis (i.e. IG and IA: Ames \& Mitchell, 1955; Hartman, 1956).

The similarities observed in the Pauly-positive imidazoles accumulated by mutants of Staphylococcus aureus and Salmonella typhimurium and the similarities observed at the genetic level (Kloos \& Pattee, 1965) support the conclusion that the pathways used for the biosynthesis of L-histidine are similar, if not identical, in both species. This conclusion is based upon the assumption that the imidazoles accumulated by $S$. aureus, like those accumulated by $S$. typhimurium, are modified intermediates of the pathway, and that, with the exception of AIC and AIC-R, the modifications are identical. Final conclusions about the pathway used in $S$. aureus, however, must await further studies to identify the specific substrates and products of the enzymes essential for the synthesis of L-histidine in this organism.

The authors express their appreciation to Dr P. E. Hartman, The Johns Hopkins University, for providing the mutants of Salmonella typhimurium and certain of the imidazoles used. This study was supported in part by Public Health Service Research Grant AI-04202 from the National Institute of Allergy and Infectious Diseases, and by Grant GB-2325 from the National Science Foundation.

\section{REFERENCES}

Ames, B. B., Garry, B. \& Herzenberg, L. (1960). The genetic control of the enzymes of histidine biosynthesis in Salmonella typhimurium. J. gen. Microbiol. 22, 369.

Ames, B. N. \& Hartman, P. E. (1962). Genes, enzymes, and control mechanisms in histidine biosynthesis. In The Molecular Basis of Neoplasia, pp. 322-45. Austin, Texas: University of Texas Press.

Ames, B. N., Martin, R. G. \& GarRy, B. J. (1961). The first step of histidine biosynthesis. J. biol. Chem. 236, 2019.

Ames, B. N. \& Mitchell, H. K. (1952). The paper chromatography of imidazoles. $J$. Amer. chem. Soc. 74, 252.

Ames, B. N. \& Mitcheld, H. K. (1955). The biosynthesis of histidine. Imidazoleglycerol phosphate, imidazoleacetolphosphate, and histidinol phosphate. J. biol. Chem. 212, 687.

Ames, B. N., Mrtchell, H. K. \& Mrtchell, M. B. (1953). Some new naturally occurring imidazoles related to the biosynthesis of histidine. J. Amer. chem. Soc. 75, 1015.

Block, R. J., Durnum, E. L. \& Zweig, G. (1958). A Manual of Paper Chromatography and Paper Electrophoresis, pp. 110-69. New York: Academic Press Inc. 
Davis, B. D. (1949). The isolation of biochemically deficient mutants of bacteria by means of penicillin. Proc. natn. Acad. Sci., U.S.A. 41, 891.

HaAs, F., Mitchell, M. B., Ames, B. N. \& Mrtchell, H. K. (1952). A series of histidineless mutants of Neurospora crassa. Genetics, 37, 217.

Hartman, P. E. (1956). Linked loci in the control of consecutive steps in the primary pathway of histidine synthesis in Salmonella typhimurium. Genetic Studies with Bacteria, Publ. Carneg. Instn. no. 612, p. 35.

Hartman, P. E., Loper, J. C. \& Serman, D. (1960). Fine structure mapping by complete transduction between histidine-requiring Salmonella mutants. J. gen. Microbiol. 22, 323.

Kroos, W. E. \& Pattee, P. A. (1965). Transduction analysis of the histidine region in Staphylococcus aureus. J. gen. Microbiol. 39, 195.

LEDERBERG, J. (1950). Isolation and characterization of biochemical mutants of bacteria. Meth. med. Res. 3, 5.

Lederberg, J. \& Lederberg, E. M. (1952). Replica plating and indirect selection of bacterial mutants. J. Bact. 63, 399.

Pattee, P. A. \& Baldwin, J. N. (1961). Transduction of resistance to chlortetracycline and novobiocin in Staphylococcus aureus. J. Bact. 82, 875.

Pattee. P. A. \& Baldwin, J. N. (1962). Transduction of resistance to some macrolide antibiotics in Staphylococcus aureus. J. Bact. 84, 1049.

Smith, D. W. E. \& Ames, B. N. (1964). Intermediates in the early steps of histidine biosynthesis. J. biol. Chem. 239, 1848.

Voget, H. J. \& BonNer, D. M. (1956). Acetylornithinase of Escherichia coli; partial purification and some properties. J. biol. Chem. 218, 97 .

Vogel, H. J., Davis, B. D. \& Mingroli, E. S. (1951). L-Histidinol, a precursor of Lhistidine in Escherichia coli. J. Amer. chem. Soc. 73, 1897. 